\title{
Arritmias en la paciente embarazada
}

\author{
Edison Muñoz Ortiz ${ }^{a, b, *}$, Juan Fernando Agudelo ${ }^{c}$, Jesús Velásquez ${ }^{\mathrm{d}, \mathrm{e}}$ \\ y Edwin F. Arévalo ${ }^{a, b}$
}

\begin{abstract}
a Clínica de embarazo y enfermedad cardiaca, Unidad Cardiovascular, Hospital Universitario San Vicente Fundación, Medellín, Colombia

b Sección de Cardiología, departamento de Medicina Interna, Universidad de Antioquia, Medellín, Colombia

c Unidad Cardiovascular, Hospital Universitario San Vicente Fundación, Medellín, Colombia

d Clínica de embarazo y enfermedad cardiaca, Departamento de Gineco-obstetricia, Hospital Universitario San Vicente Fundación, Medellín, Colombia

e Sección perinatología y alto riesgo obstétrico, departamento de Obstetricia y ginecología, Universidad de Antioquia, Medellín, Colombia
\end{abstract}

Recibido el 4 de septiembre de 2016; aceptado el 16 de noviembre de 2016 Disponible en Internet el 2 de febrero de 2017

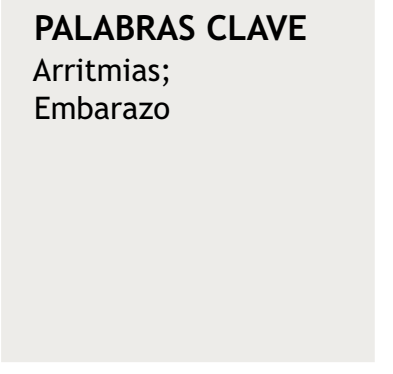

KEYWORDS

Arrhythmias;

Pregnancy
Resumen Las arritmias cardiacas son complicaciones frecuentes en el embarazo e incluso durante éste aumenta su incidencia, siendo más frecuentes las supraventriculares que las ventriculares. Las arritmias en la paciente embarazada representan además un riesgo para presentar eventos fetales adversos tanto por la arritmia en sí misma, como por los riesgos potenciales de los medicamentos usados para el tratamiento. En este artículo se revisan los aspectos más relevantes respecto al mecanismo y tratamiento de las arritmias en la paciente embarazada. (c) 2016 Sociedad Colombiana de Cardiología y Cirugía Cardiovascular. Publicado por Elsevier España, S.L.U. Este es un artículo Open Access bajo la licencia CC BY-NC-ND (http:// creativecommons.org/licenses/by-nc-nd/4.0/).

\section{Arrhythmias in pregnant patients}

Abstract Cardiac arrhythmias are frequent complications in pregnancy and their incidence even increases during this period, supraventricular being more common than ventricular ones. Arrhythmias in pregnancy also entail a risk to develop fetal adverse effects due to the arrhythmia itself, as well as due to the potential risks of drugs used for their treatment. This article reviews the most relevant aspects regarding the mechanism and treatment of arrhythmias in pregnant patients.

(C) 2016 Sociedad Colombiana de Cardiología y Cirugía Cardiovascular. Published by Elsevier España, S.L.U. This is an open access article under the CC BY-NC-ND license (http:// creativecommons.org/licenses/by-nc-nd/4.0/).

\footnotetext{
* Autor para correspondencia.

Correo electrónico: edisonhoy@yahoo.es (E. Muñoz Ortiz).
} 


\section{Introducción}

Las arritmias cardiacas son una complicación frecuente durante el embarazo; de hecho se ha considerado que éste incrementa la incidencia de arritmias en pacientes con y sin enfermedad cardiaca estructural ${ }^{1-4}$. Esto se demuestra cuando se evidencia que el riesgo de una embarazada de presentar taquicardia supraventricular comparada con población no gestante se ha calculado en $5.1^{5}$. Sin embargo, en la mayoría de casos las palpitaciones y arritmias más reportadas son benignas y no requieren tratamiento, pero se debe tener presente que en otros las arritmias pueden ser un problema clínico considerable.

Las arritmias supraventriculares son las más comunes y ocurren de forma sostenida hasta en 1,3\% de las mujeres embarazadas sin enfermedad cardiaca estructural, de las cuales el $34 \%$ tienen el primer episodio de la arritmia durante el embarazo y 29 a $44 \%$ tienen una exacerbación de arritmias ya conocidas ${ }^{6}$. En nuestra clínica de embarazadas con enfermedad cardiaca, en promedio $22 \%$ de las evaluaciones se deben a algún tipo de arritmia, de las cuales $87,5 \%$ son supraventriculares y el resto ventriculares (datos no publicados).

Adicionalmente, hasta en $20 \%$ de los embarazos puede haber eventos fetales adversos, tales como prematuridad, síndrome de dificultad respiratoria y feto pequeño para la edad gestacional ${ }^{3}$.

El tratamiento agudo de la arritmia suele hacerse con medicamentos como adenosina, metoprolol o cardioversión eléctrica, debido a que se consideran seguros para el feto $y$, en general, logran abortar el evento arrítmico; mientras tanto, la ablación por radiofrecuencia, que es un procedimiento curativo de la arritmia en muchos casos, raramente se lleva a cabo durante el embarazo pues la radiación debida a la exposición a rayos $x$ también puede ser dañina para el feto; pese a ello, en quienes los medicamentos no han sido efectivos para terminar una arritmia aguda o prevenir recurrencias, el procedimiento de ablación puede ser la única alternativa terapéutica ${ }^{6}$.

El tratamiento de las diferentes arritmias durante la gestación es un aspecto de la cardiología no muy bien definido debido a las particularidades que tienen estas pacientes, dadas por los cambios fisiológicos del embarazo y la presencia del feto que limitan muchas intervenciones. Los tratamientos antiarritmicos no solo pueden ser dañinos para el feto, sino que además tienen pocos estudios en humanos, hecho que circunscribe las recomendaciones en guías internacionales a aspectos muy generales ${ }^{7}$.

En este artículo se revisan los aspectos más relevantes de las arritmias en la paciente embarazada.

\section{Mecanismos arritmogénicos en el embarazo}

La explicación fisiopatológica de porqué las arritmias aumentan durante el embarazo no es claro, pero se ha considerado la consecuencia de la combinación de cambios hemodinámicos, hormonales y autonómicos.

Los cambios hemodinámicos del embarazo incluyen un incremento en el volumen sanguíneo efectivo circulante de 30 a $50 \%$ a partir de las 8 semanas de gestación, con su mayor efecto alrededor de las 34 semanas; además, el gasto
Tabla 1 Definición de categorías de riesgo fetal por medicamentos en el embarazo

Categoría en el Definición
embarazo

A

Estudios controlados no muestran riesgo. Estudios bien controlados en mujeres embarazadas no han demostrado riesgo para el feto en cualquier trimestre del embarazo

B No evidencia de riesgo en humanos. Estudios bien controlados en mujeres embarazadas no han mostrado incremento del riesgo de anormalidades fetales luego de hallazgos adversos en animales, o en ausencia de estudios adecuados en humanos, estudios en animales no muestran riesgo fetal. El riesgo de daño fetal es remoto pero permanece como una posibilidad

C Riesgo no puede ser excluido. No hay estudios bien controlados en humanos y estudios en animales han demostrado un riesgo para el feto, o tampoco se tienen esos estudios. Hay posibilidad de daño fetal si se administra durante el embarazo, pero puede usarse si el potencial beneficio supera los riesgos

D Evidencia de riesgo. Estudios en humanos han mostrado evidencia de daño fetal. Sin embargo, podría usarse si el potencial beneficio de su uso en la mujer embarazada sobrepasa el riesgo

X Contraindicado en el embarazo. Estudios en animales o humanos han mostrado riesgo fetal, lo cual claramente sobrepasa cualquier posible beneficio en el paciente

cardiaco también aumenta, secundario al incremento en el volumen latido del $35 \%$, así como de la frecuencia cardiaca en un $15 \%^{8}$. El aumento del volumen plasmático se ha implicado en la producción de estiramiento de los miocitos auriculares y ventriculares, que podrían causar posdespolarizaciones tempranas, conducción lenta, acortamiento del periodo refractario y dispersión espacial a través de canales iónicos activados por el estiramiento $0^{9,10}$. Adicionalmente, la dilatación de las fibras miocárdicas y el aumento del tamaño cardiaco, podrían hacer más fácil sostener los circuitos de reentrada al aumentar la longitud del trayecto implicado en el fenómeno de reentrada ${ }^{8}$. Adicionalmente, el aumento de la frecuencia cardiaca en reposo, más acentuado en el tercer trimestre del embarazo, también se ha implicado como predictor de arritmogénesis ${ }^{11}$.

En cuanto a los cambios hormonales y autonómicos, se ha descrito en estudios animales y en algunos reportes en 
Tabla 2 Recomendaciones para uso de antiarrítmicos en el embarazo

\begin{tabular}{|c|c|c|c|c|c|c|}
\hline Medicamento & $\begin{array}{l}\text { Clase } \\
\text { Vaugham- } \\
\text { Williams }\end{array}$ & $\begin{array}{l}\text { Categoría } \\
\text { FDA }\end{array}$ & $\begin{array}{l}\text { Efectos adversos } \\
\text { potenciales }\end{array}$ & $\begin{array}{l}\text { Indicación primaria } \\
\text { en la madre }\end{array}$ & $\begin{array}{l}\text { Uso durante la } \\
\text { lactancia }\end{array}$ & Observaciones \\
\hline Lidocaína & IB & B & $\begin{array}{l}\text { Efectos adversos en } \\
\text { SNC, bradicardia }\end{array}$ & $\begin{array}{l}\text { TV, arritmias } \\
\text { debidas a toxicidad } \\
\text { por digoxina }\end{array}$ & Puede usarse & $\begin{array}{l}\text { Larga historia de } \\
\text { seguridad. Evitar en } \\
\text { sufrimiento fetal }\end{array}$ \\
\hline Propafenona & IC & $\mathrm{C}$ & $\begin{array}{l}\text { Incremento en } \\
\text { mortalidad en } \\
\text { pacientes con } \\
\text { previo IAM. Leve } \\
\text { efecto } \\
\text { betabloqueador }\end{array}$ & TSV y TV & Desconocido & $\begin{array}{l}\text { Pocos datos } \\
\text { disponibles }\end{array}$ \\
\hline Betabloqueadores & ॥ & $C / D$ & $\begin{array}{l}\text { Retardo del } \\
\text { crecimiento } \\
\text { intrauterino, } \\
\text { bradicardia fetal, } \\
\text { hipoglicemia, apnea } \\
\text { fetal }\end{array}$ & $\begin{array}{l}\text { TSV, TV idiopática, } \\
\text { control de } \\
\text { respuesta } \\
\text { ventricular en FA }\end{array}$ & $\begin{array}{l}\text { Evitar atenolol. } \\
\text { Metoprolol y } \\
\text { propranolol pueden } \\
\text { usarse }\end{array}$ & $\begin{array}{l}\text { Generalmente bien } \\
\text { tolerados, evitar } \\
\text { durante el primer } \\
\text { trimestre si es } \\
\text { posible, preferir los } \\
\text { cardioselectivos }\end{array}$ \\
\hline Amiodarona & III & $\mathrm{D}$ & $\begin{array}{l}\text { Hipotiroidismo } \\
\text { fetal, } \\
\text { prematuridad, bajo } \\
\text { peso al nacer, } \\
\text { malformaciones } \\
\text { congénitas }\end{array}$ & $\begin{array}{l}\text { Arritmias } \\
\text { ventriculares } \\
\text { amenazantes } \\
\text { para la vida }\end{array}$ & Evitar & $\begin{array}{l}\text { Evitar si es posible, } \\
\text { especialmente } \\
\text { durante el primer } \\
\text { trimestre }\end{array}$ \\
\hline Dronedarona & III & $x$ & Puntas torcidas & FA & Contraindicado & Contraindicado \\
\hline Verapamilo & IV & C & $\begin{array}{l}\text { Hipotensión } \\
\text { materna, bloqueo } \\
\text { cardiaco y } \\
\text { bradicardia fetal }\end{array}$ & $\begin{array}{l}\text { TSV, TV idiopática, } \\
\text { control de } \\
\text { respuesta } \\
\text { ventricular en FA }\end{array}$ & Puede usarse & $\begin{array}{l}\text { Relativamente bien } \\
\text { tolerado, pero } \\
\text { pueden preferirse } \\
\text { opciones más } \\
\text { seguras }\end{array}$ \\
\hline Diltiazem & IV & C & $\begin{array}{l}\text { Datos limitados. } \\
\text { Similar a } \\
\text { verapamilo }\end{array}$ & $\begin{array}{l}\text { TSV, control de } \\
\text { respuesta } \\
\text { ventricular en FA }\end{array}$ & Puede usarse & $\begin{array}{l}\text { Preferido el } \\
\text { verapamilo por } \\
\text { mayor experiencia }\end{array}$ \\
\hline Adenosina & No aplica & C & $\begin{array}{l}\text { Disnea y bradicardia } \\
\text { materna }\end{array}$ & $\begin{array}{l}\text { Terminación aguda } \\
\text { de TSV }\end{array}$ & $\begin{array}{l}\text { Desconocido, pero } \\
\text { probablemente bien } \\
\text { tolerada }\end{array}$ & $\begin{array}{l}\text { Primera opción para } \\
\text { terminación de TSV } \\
\text { que dependan del } \\
\text { nodo AV }\end{array}$ \\
\hline Digoxina & No aplica & C & Bajo peso al nacer & $\begin{array}{l}\text { TSV, control de } \\
\text { respuesta } \\
\text { ventricular en FA }\end{array}$ & Puede usarse & $\begin{array}{l}\text { Gran experiencia } \\
\text { en su uso }\end{array}$ \\
\hline
\end{tabular}

Modificada de: Enriquez A, Economy KE, Tedrow UB. Contemporary management of arrhythmias during pregnancy. Circ Arrhythm Electrophysiol. 2014;7:961-7. FA: fibrilación auricular; IAM: infarto agudo de miocardio; SNC: sistema nervioso central; TSV: taquicardia supraventricular; TV: taquicardia ventricular.

humanos, que el estradiol y la progesterona pueden aumentar la aparición de arritmias, fenómeno que podría relacionarse especialmente con los estrógenos, por su potencial para aumentar receptores adrenérgicos ${ }^{8}$.

Estos mecanismos arritmogénicos, además de llevar a la mayor frecuencia de arritmias en el embarazo, perjudican a la madre y conllevan riesgos adicionales para el feto, pues las arritmias pueden hacer que la presión arterial sea más baja de lo habitual y, por ende, reducir la perfusión placentaria ${ }^{12}$. Dentro de los efectos adversos fetales se describen la prematuridad (con las complicaciones que implica), síndrome de dificultad respiratoria, feto pequeño para la edad gestacional e incluso enfermedades cardiacas congénitas; muchos de los casos reportados con estos efectos adversos, tuvieron tratamiento con antiarritmicos durante el embarazo, o uso de anticoagulantes, lo que hace difícil diferenciar entre el impacto aislado de la arritmia o el efecto de los medicamentos usados en forma concomitante ${ }^{6}$.

\section{Medicamentos antiarrítmicos}

En términos generales, los antiarrítmicos se consideran categoría C y D por la Administración de Alimentos y Medicamentos (FDA, su sigla en inglés). Se debe tener presente que los antiarritmicos con un riesgo aceptable y que logran ser efectivos en el tratamiento de las arritmias maternas previas al embarazo o durante el embarazo pueden continuarse, pues al lograr estabilizar el trastorno del ritmo, podrían ser más los beneficios que los riesgos al mantener la terapia, si bien lo ideal es buscar una terapia con la dosis mínima efectiva ${ }^{1,6,13-15}$.

En la tabla 1 se explican las categorías de los medicamentos según la FDA y en la tabla 2 se resumen los medicamentos 
antirrítmicos de los que se dispone en nuestro medio, se describe la clase de medicamento antiarrítmico, la categoría de la FDA y sus efectos materno-fetales y recomendaciones de uso durante el embarazo y la lactancia.

\section{Cardioversión eléctrica}

La cardioversión eléctrica es una estrategia de tratamiento adecuada y segura en todas las etapas del embarazo, aunque tiene un riesgo teórico de inicio de parto pretérmino en etapas avanzadas. Esta intervención se considera la terapia de elección en las pacientes con inestabilidad hemodinámica, mientras que puede ser una alternativa electiva en arritmias sin respuesta a terapia médica ${ }^{13,15}$. No altera el flujo sanguíneo al feto, y además una muy pequeña cantidad de energía le alcanza, de modo que el riesgo de arritmias fetales es mínimo ${ }^{13,16}$; sin embargo, hay reportes de casos de necesidad de cesárea urgente por arritmias fetales después de una cardioversión, y por tanto, es necesario monitorizar al feto ${ }^{17}$.

\section{Ablación con catéter}

Es una estrategia muy efectiva para el tratamiento de las arritmias y está recomendada en guías de manejo de arritmias supraventriculares y ventriculares ${ }^{1,18}$. Sin embargo, en lo que respecta a pacientes embarazadas hay pocos estudios, y suele no usarse debido al riesgo de radiación por los rayos $x$ empleados en la fluoroscopia ${ }^{12,13}$. A pesar de lo anterior, puede hacerse de emergencia ante la falta de respuesta a la terapia médica e incluso a la cardioversión eléctrica repetida, aunque por fortuna estos casos son la excepción. En los casos reportados, los tiempos de fluoroscopia van de 0 segundos a 36 minutos $^{19-21}$, sin complicaciones en el procedimiento o tardías; obviamente se deberá tener presente que puede haber sesgo de publicación de los casos con resultados adversos, adicional a que el feto podría tener riesgos futuros de cáncer ${ }^{6,22}$. Aunque esos efectos adversos pueden reducirse con la protección contra la radiación, el uso del mínimo tiempo de fluoroscopia posible o el empleo de técnicas alternativas como los sistemas electroanatómicos $3 \mathrm{D}$ que minimicen aún más el uso de radiación o incluso eviten completamente la exposición a radiación, tienen resultados adecuados tanto para la madre como para el feto ${ }^{23-27}$ y podrían ser una alternativa de terapia con menos riesgos de los habituales, al llevar a un riesgo de daño fetal mínimo; se debe seguir considerando un procedimiento a usarse solo en mujeres embarazadas con arritmias severas sin respuesta a la terapia médica ${ }^{6,13-15}$.

\section{Recomendaciones de guías internacionales}

Las guías recientes de manejo de arritmias ventriculares de la Sociedad europea de cardiología ${ }^{18}$ y de arritmias supraventriculares de la Sociedad americana del corazón ${ }^{1}$, hacen las siguientes recomendaciones para el manejo de pacientes embarazadas con arritmias, a las cuales se adhieren los autores de este documento, aclarándose que no se incluyeron las recomendaciones de medicamentos no disponibles en nuestro medio.

\section{Recomendaciones en arritmias ventriculares ${ }^{18}$}

- Se recomienda el implante de un cardiodesfibrilador si surge una indicación durante el embarazo (clase ।, nivel de evidencia $\mathrm{C}$ ).

- Se recomienda betabloqueadores durante el embarazo y periodo posparto en pacientes con síndrome de QT largo o taquicardia ventricular polimórfica catecolaminérgica (clase I, nivel de evidencia C).

- Se recomienda metoprolol oral, propranolol o verapamilo para el manejo a largo término de taquicardia ventricular sostenida idiopática (clase ı, nivel de evidencia C).

- Se recomienda la cardioversión eléctrica inmediata para taquicardia ventricular sostenida, especialmente si hay inestabilidad hemodinámica (clase ।, nivel de evidencia C).

- Se debería considerar amiodarona intravenosa para la cardioversión de taquicardia ventricular monomórfica sostenida cuando la paciente está hemodinámicamente inestable y es refractaria a cardioversión eléctrica o no responde a otros medicamentos (clase Ila, nivel de evidencia C).

- Se puede considerar la ablación con catéter para el manejo de taquicardias refractarias a medicamentos o pobremente tolerados (clase IIb, nivel de evidencia C).

\section{Recomendaciones para el manejo de arritmias supraventriculares (excluida la fibrilación auricular $)^{1}$}

- Se recomiendan las maniobras vagales para el tratamiento agudo de pacientes embarazadas con taquicardias supraventriculares (clase I, nivel de evidencia C).

- Se recomienda la adenosina para el tratamiento agudo en pacientes embarazadas con taquicardia supraventricular (clase I, nivel de evidencia C).

- Se recomienda la cardioversión sincronizada para el tratamiento agudo en pacientes embarazadas con taquicardia supraventricular e inestabilidad hemodinámica, cuando la terapia farmacológica no es efectiva o está contraindicada (clase I, nivel de evidencia C)

- El metoprolol intravenoso o el propranolol son razonables para el tratamiento agudo en pacientes embarazadas con taquicardia supraventricular, cuando la adenosina no es efectiva o está contraindicada (clase lla, nivel de evidencia C).

- Puede considerarse la administración de amiodarona intravenosa para el tratamiento agudo en pacientes embarazadas con taquicardia supraventricular potencialmente amenazante para la vida, cuando otras terapias no son efectivas o están contraindicadas (clase Ilb, nivel de evidencia C).

- Entre los medicamentos que pueden utilizarse solos o en combinación para el manejo continuo de arritmias supraventriculares en pacientes embarazadas se incluyen: digoxina, metoprolol, propafenona, propranolol y verapamilo (clase Ila, nivel de evidencia C).

- Puede ser razonable la ablación con catéter en pacientes embarazadas con taquicardia supraventricular altamente sintomática, recurrente y refractaria a medicamentos, 


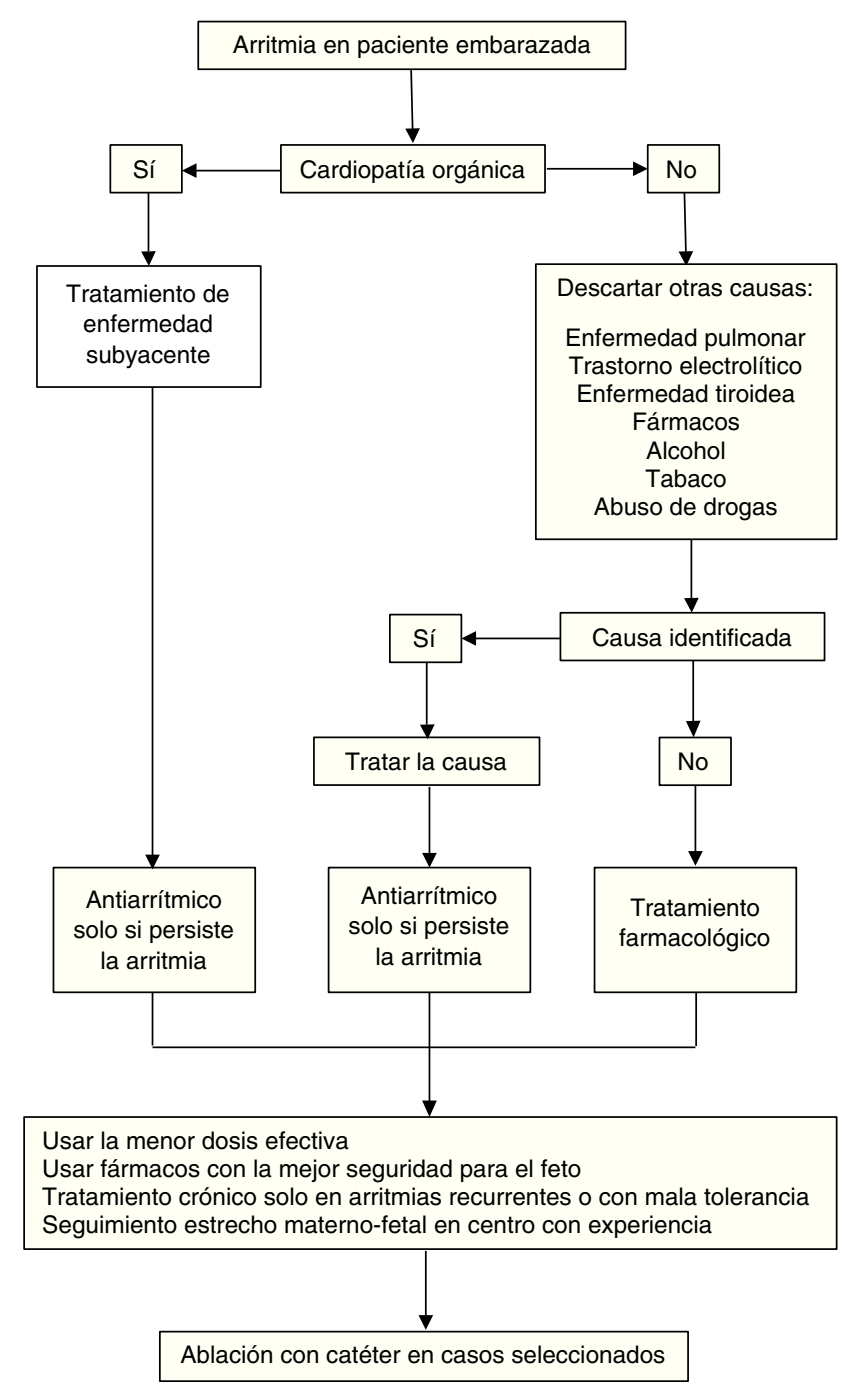

Figura 1 Algoritmo para el tratamiento crónico de arritmias en el embarazo. Modificado de: Alberca T, Palma J, Garcia-Cosio F. Arritmias y embarazo. Rev Esp Cardiol. 1997;50:749-59.

con esfuerzos en reducir al mínimo la exposición a la radiación (clase IIb, nivel de evidencia $\mathrm{C}$ )

- Se puede considerar amiodarona oral para el manejo continuo de pacientes embarazadas con arritmias supraventriculares altamente sintomáticas, recurrentes, cuando otros medicamentos no son efectivos o están contraindicados (clase Ilb, nivel de evidencia C).

Finalmente, y teniendo en cuenta todo lo discutido, la figura 1 constituye una propuesta de algoritmo de tratamiento crónico de las arritmias en la paciente embarazada ${ }^{28}$.

\section{Responsabilidades éticas}

Protección de personas y animales. Los autores declaran que para esta investigación no se han realizado experimentos en seres humanos ni en animales.
Confidencialidad de los datos. Los autores declaran que en este artículo no aparecen datos de pacientes.

Derecho a la privacidad y consentimiento informado. Los autores declaran que en este artículo no aparecen datos de pacientes.

\section{Financiación}

No requirió financiación.

\section{Conflicto de intereses}

Los autores declaran no tener conflicto de intereses.

\section{Bibliografía}

1. Page RL, Joglar JA, Caldwell MA, Calkins H, Conti JB, Deal BJ, et al. 2015 ACC/AHA/HRS guideline for the management of adult patients with supraventricular tachycardia: A report of the American college of cardiology/American heart association task force on clinical practice guidelines and the heart rhytm society. J Am Coll Cardiol. 2015 [Epub ahead of print].

2. Siu SC, Sermer M, Colman JM, Alvarez AN, Mercier LA, Morton $B C$, et al. Cardiac Disease in Pregnancy (CARPREG) Investigators. Prospective multicenter study of pregnancy outcomes in women with heart disease. Circulation. 2001;104:515-21.

3. Silversides CK, Harris L, Haberer K, Sermer M, Colman JM, Siu SC. Recurrence rates of arrhythmias during pregnancy in women with previous tachyarrhythmia and impact on fetal and neonatal outcomes. Am J Cardiol. 2006;97:1206-12.

4. Shotan A, Ostrzega E, Mehra A, Johnson JV, Elkayam U. Incidence of arrhythmias in normal pregnancy and relation to palpitations, dizziness, and syncope. Am J Cardiol. 1997;79:1061-4.

5. Tawam M, Levine J, Mendelson M, Goldberger J, Dyer A, Kadish A. Effect of pregnancy on paroxysmal supraventricular tachycardia. Am J Cardiol. 1993;72:838-40.

6. Szumowski L, Szufladowicz E, Orczykowski M, Bodalski R, Derejko P, Przybylski A, et al. Ablation of severe drug-resistant tachyarrhythmia during pregnancy. J Cardiovasc Electrophysiology. 2010;21:877-82.

7. Regitz-Zagrosek V, Lundqvist CB, Borghi C, Cifkova R, Ferreira $\mathrm{R}$, Foidart JM, et al. ESC guidelines on the management of cardiovascular diseases during pregnancy. The task force on the manegment of cardiovascular diseases during pregnancy of the European society of cardiology (ESC). Eur Heart J. 2011;32:3147-97.

8. Enriquez A, Economy KE, Tedrow UB. Contemporary management of arrhythmias during pregnancy. Circ Arrhythm Electrophysiol. 2014;7:961-7.

9. Franz MR, Cima R, Wang D, Profitt D, Kurz R. Electrophysiological effects of myocardial stretch and mechanical determinants of stretch-activated arrhythmias. Circulation. 1992;86:968-78.

10. Ninio DM, Saint DA. The role of stretch-activated channels in atrial fibrillation and the impact of intracellular acidosis. Prog Biophys Mol Biol. 2008;97:401-6.

11. Soliman EZ, Elsalam MA, Li Y. The relationship between high resting heart rate and ventricular arrhythmogenesis in patients referred to ambulatory $24 \mathrm{~h}$ electrocardiographic recording. Europace. 2010;12:261-5.

12. Bigelow AM, Crane SS, Khoury FR, Clark JM. Catheter ablation of supraventricular tachycardia without fluoroscopy during pregnancy. Obstet Gynecol. 2015;125:1338-41. 
13. Bartalena L, Bogazzi F, Braverman LE, Martino E. Effects of amiodarone administration during pregnancy on neonatal thyroid function and subsequent neurodevelopment. J Endocrinol Invest. 2001;24:116-30.

14. Joglar JA, Page RL. Management of arrhythmia syndromes during pregnancy. Curr Opin Cardiol. 2014;29:36-44.

15. Knotts RJ, Garan H. Cardiac arrhythmias in pregnancy. Sem Perinatol. 2014;38:285-8.

16. Wang YC, Chen CH, Su HY, Yu MH. The impact of maternal cardioversión on fetal haemodynamics. Eur J Obstet Gynecol Reprod Biol. 2006;126:268-9.

17. Barnes EJ, Eben F, Patterson D. Direct current cardioversion during pregnancy should be performed with facilities available for fetal monitoring and emergency caesarean section. BJOG. 2002;109:1406-7.

18. Priori SG, Blomström-Lundqvist C, Mazzanti A, Blom N, Borggrefe M, Camm J, et al. 2015 ESC guidelines for the management of patients with ventricular arrhythmias and the prevention of sudden cardiac death. The task force for the management of patients with ventricular arrhythmias and the prevention of sudden cardiac death of the European society of cardiology (ESC). Eur Heart J. 2015;36:2793-867.

19. Bombelli F, Lagona F, Salvati A, Catalfamo L, Ferrari AG, Pappone C. Radiofrequency catheter ablation in drug refractory maternal supraventricular tachycardias in advanced pregnancy. Obstet Gynecol. 2003;102(Part 2):1171-3.

20. Berruezo A, Diez GR, Berne P, Esteban M, Mont L, Brugada J. Low exposure radiation with conventional guided radiofrequency catheter ablation in pregnant women. Pacing Clin Electrophysiol. 2007;10:1299-302.
21. Bongiorni MG, Di Cori A, Soldati E, Zucchelli G, Segreti L, Solarino G, et al. Radiofrequency catheter ablation of atrioventricular nodal reciprocating tachycardia using intracardiac echocardiography in pregnancy. Europace. 2008;10: 1018-21.

22. Wakeford R, Little MP. Risk coefficients for childhood cancer after intrauterine irradiation: a review. Int J Radiat Biol. 2003;79:293-309.

23. Yang PS, Park J, Pak HN. Radiofrequency catheter ablation of incessant atrial tachycardia in pregnant women with minimal radiation exposure: Lessons from two case studies. J Cardiol Cases. 2014;9:213-6.

24. Stec S, Krynski T, Baran J, Kulakowski P. Rescue ablation of electrical storm in arrhythmogenic right ventricular cardiomyopathy in pregnancy. BMC Cardiovasc Disord. 2013;13:58.

25. Manjaly ZR, Sachdev B, Webb T, Rajappan K. Ablation of arrhythmia in pregnancy can be done safely when necessary. Eur J Obstet Gynecol Reprod Biol. 2011;157:116-7.

26. Raman AS, Sharma S, Hariharan R. Minimal use of fluoroscopy to reduce fetal radiation exposure during radiofrequency catheter ablation of maternal supraventricular tachycardia. Texas Hear Inst J. 2015;42:152-4.

27. Wu H, Ling LH, Lee G, Kistler PM. Successful catheter ablation of incessant atrial tachycardia in pregnancy using three-dimensional electroanatomical mapping with minimal radiation. Intern Med J. 2012;42:709-12.

28. Alberca T, Palma J, Garcia-Cosio F. Arritmias y embarazo. Rev Esp Cardiol. 1997;50:749-59. 